\section{Evaluating clinical signs in trabeculectomized eyes}

JG Crowston ${ }^{1,2}$, JF Kirwan ${ }^{1,3}$, A Wells ${ }^{1}$, C Kennedy ${ }^{3}$ and IE Murdoch ${ }^{1,3}$

$(\kappa=0.56 \pm 0.19)$, and fair for bleb morphology $(\kappa=0.31 \pm 0.12)$. Microcysts were not reliably detected using either technique.

Conclusion SL biomicroscopy and TM telemedicine examination may permit reliable clinical assessment of trabeculectomized eyes. However, remote examination with TM is more limited with respect to assessing bleb height and bleb wall thickness. The assessment of bleb morphology and microcysts was unreliable with both instruments. We propose that TM examination of trabeculectomized eyes appears safe and appropriate in situations where face-to-face examination by an ophthalmologist is not practical.

Eye (2004) 18, 299-303. doi:10.1038/

sj.eye. 6700638

Keywords: telemedicine; glaucoma; bleb; trabeculectomy; interobserver agreement study

\section{Introduction}

Postoperative management of glaucomatous eyes following trabeculectomy is labour intensive. The use of releasable or adjustable sutures, bleb needling, and injection of antiscarring agents may provide the ophthalmologist with the opportunity to modify the outcome of filtering surgery. A number of clinical signs have been associated with bleb failure because of subconjunctival scar formation. ${ }^{1-4}$ In addition, the use of antimetabolites, such as mitomycin-C, can alter bleb morphology to produce thin bleb walls with avascular, hypocellular tissue, ${ }^{5,6}$ which may predispose to postoperative complications such as bleb leak and endophthalmitis. ${ }^{7,8}$ These factors highlight the need for careful examination of the filtration site. However, a lack of information exists as to the levels of agreement between ophthalmologists with
${ }^{1}$ Glaucoma Unit Moorfields Eye Hospital London, UK

${ }^{2}$ University Eye Clinic Westmead Hospital Westmead, Sydney NSW, Australia

${ }^{3}$ Department of Epidemiology and International Eye Health Institute of Ophthalmology London, UK

Correspondence:

IE Murdoch

Department of Epidemiology and International Eye Health Institute of Ophthalmology 11-43 Bath Street London EC1V 9EL, UK Tel: + 442076086896 Fax: +44 2072503207 E-mail: IEMurdoch@ compuserve.com

Received: 20 December 2002

Accepted: 6 May 2003

The authors have no proprietary interest in any of the devices described in the paper 
respect to detecting clinical signs in trabeculectomized eyes.

Frequent postoperative assessment after surgery is not practical in all health care settings, and remote examination using telemedicine (TM) may be of use. The quality of information delivered to the remote ophthalmologist, however, should be of adequate quality. TM technologies are becoming an integral part of ophthalmic clinical practice and teaching. ${ }^{9}$ Preliminary studies suggest that this mode of remote consultation is both cost-effective and acceptable to patients. ${ }^{10}$ A paucity of information, however, exists as to how TM fares as a tool for examining specific clinical entities compared to face-to-face slit-lamp (SL) biomicroscopy. The aim of this study was to assess the interobserver agreement and the variability of clinical signs in trabeculectomized eyes when evaluated by SL biomicroscopy or remotely by TM.

\section{Method}

\section{Study design}

A prospective interobserver agreement study was performed with 40 eyes of 40 patients that had previously undergone glaucoma filtration surgery. Local ethics committee approval was granted prior to initiation of this study (JC1001). Patients were recruited from the Glaucoma Service at Moorfields Eye Hospital. Each eye was examined separately by three ophthalmologists, two using SL biomicroscopy and one remotely by TM.

Examination technique was determined on a rotational basis with the order determined by randomization. The same three examiners were employed for all six assessment sessions. In each session, one examiner performed the remote examination in an adjacent building for the whole session. The remaining two examiners alternated between performing the first and second SL examination. Real-time SL video images were transferred using a $384 \mathrm{kbps}$ Sony 5100

videoconferencing system as shown in Figure 1.

\section{Outcome measures}

A system of bleb assessment was devised by the authors, as outlined in Table 1. An initial pilot study of a series of 15 bleb photographs was performed to test the system of assessment and train the ophthalmologists involved (data not shown). Standard bleb photographs were employed as references for bleb vascularity. As bleb vascularity was frequently not uniform across the bleb, vascularity scores were obtained for three separate areas (bleb centre, bleb edge, and nonbleb conjunctiva as shown in Figure 2). Each ophthalmologist recorded scores independently. The ophthalmologist allocated to

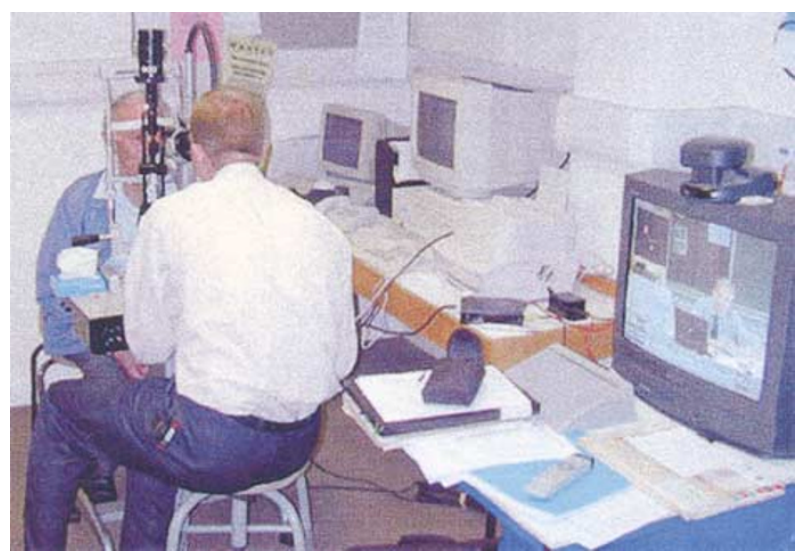

Figure 1 Live real-time video images are transferred along three ISDN lines. The remote examiner (screen) is able to switch between preset views to communicate with the SL operative and/or patient and family or to view images captured with the SL video camera.

Table 1 Grading system employed

Bleb features

Vascularity

Wall thickness

Height

Microcysts Morphology

Bleb leak

Nonbleb features

Anterior chamber \% depth of fellow eye depth

Choroidal

detachment

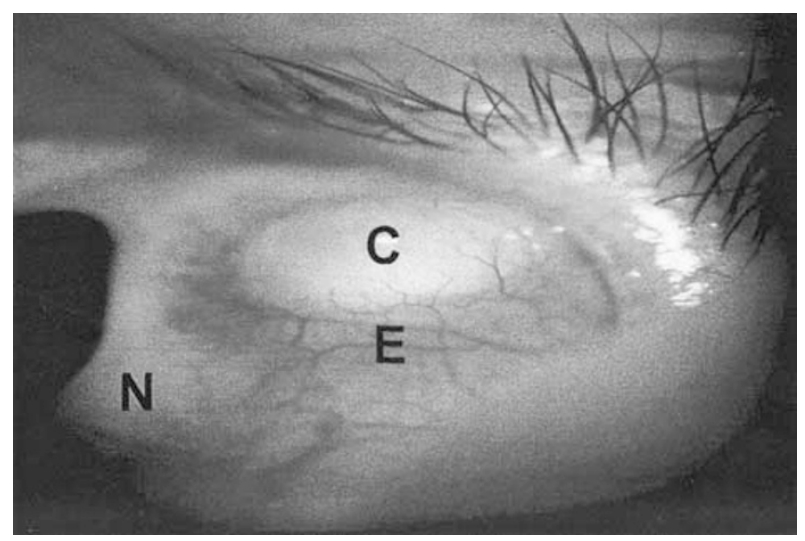

Figure 2 Conjunctival vascularity was scored for three separate zones: the central bleb area $(\mathrm{C})$, the bleb edge $(\mathrm{E})$, or nonbleb conjunctiva $(\mathrm{N})$. 
the first SL examination was followed by the second SL examiner who then operated the SL to transfer images under instructions from the third ophthalmologist viewing images remotely.

To examine for bleb leak, $2 \%$ fluorescein was applied and the eye examined with cobalt blue light with or without external digital pressure being applied to the globe.

\section{Statistical analysis}

Analysis was performed using Prism and Statmate (Graphpad, Chicago, IL, USA) to compare the findings between observers ( $\mathrm{A}$ vs $\mathrm{B}, \mathrm{A}$ vs $\mathrm{C}, \mathrm{B}$ vs $\mathrm{C}$ ) and examination instrument (SL/SL and SL/TM). For comparisons between SL and TM, the first SL exam was compared to the TM exam as the second SL examiner manoeuvred the SL for the remote examiner, and therefore these two examiners were not independent.

For bleb vascularity and anterior chamber depth, bias was assessed by comparing mean values with a paired t-test. Test variability was evaluated by assessing Bland Altman plots, and the f-distribution was used to compare 95\% limits of agreement. Weighted $\kappa$ statistics were calculated for clinical signs with nominal end points using standard definitions. ${ }^{11}$

\section{Results}

A total of 40 eyes of 40 patients were examined. The study population consisted of 16 female and 24 male patients. The time from trabeculectomy to examination ranged from 1 day to 15 years (median 5.4 years). Results of slit lamp vs slit lamp (SL/SL) and slit lamp vs telemedicine (SL/TM) are summarized in Table 2.

\section{Vascularity}

Comparison of paired SL lamp examinations (SL/SL) for overall vascularity scores showed very similar values (mean difference $0.05,95 \% \mathrm{CI}-0.19$ to 0.29 ) with similar levels of agreement for all three bleb zones. The observers agreed within one vascularity score for $75-80 \%$ of eyes. Between the first SL examination and the TM examination (SL/TM), the mean difference in vascularity scores suggested that TM may possibly over-read bleb vascularity (mean difference 0.26 units, $95 \% \mathrm{CI}-0.015$ to $0.53 ; P=0.063)$. The spread of disagreement in vascularity scores tended to be broader with SL/TM compared to SL/SL (95\% limits of agreement 2.57 vs 2.98; $P=0.054)$. Subanalysis of the three different vascularity zones revealed consistent levels of agreement for all three zones. Raters agreed within \pm 1 vascularity score for a median of $78 \%$ of eyes (range $62.5-85 \%$ ) with no difference in the level of agreement for any of the three zones. Bland-Altman plots suggested that the spread of agreement was similar across the range of anterior chamber depths.

\section{Anterior chamber depth}

Use of the fellow eye as a means of assessing anterior chamber depth provides a reproducible (but arbitrary) value. SL examinations showed good agreement for anterior chamber depth (mean difference $0.54 \%, 95 \%$ CI -6.0 to $4.9 \%$ ). Observers agreed within $10 \%$ of anterior chamber depth in $68 \%$ of eyes. A difference in estimate of greater than $50 \%$ of anterior chamber depth was observed in $8 \%$ of eyes.

Overall, anterior chamber depth estimates derived using TM were very similar (mean difference $0.81 \%, 95 \%$ CI -7.2 to $+5.6 \%$ ). The spread of disagreement between anterior chamber depth scores may be wider for the $\mathrm{SL} / \mathrm{TM}$ analysis compared to SL/SL $(P=0.087)$. Total agreement (within 10\%) was observed in $51 \%$ of eyes compared to $68 \%$ for paired SL analysis $(P=0.68)$. Bland-Altman plots suggested that spread of agreement was similar across the range of anterior chamber depths.

\section{Bleb characteristics}

Bleb height, bleb wall thickness, bleb morphology, the presence or absence of leak, and microcysts were graded according to the scales shown in Table 1.

For wall thickness, agreement was 'good' (weighted $\kappa \pm$ standard error $=0.63 \pm 0.08)$ with SL/SL and fair with $\mathrm{SL} / \mathrm{TM}(\kappa=0.39 \pm 0.13)$. Bleb height agreement was 'good' with SL/SL $(\kappa=0.67 \pm 0.1)$ and 'poor' for SL/TM $(\kappa=0.17 \pm 0.12)$. For bleb morphology, agreement was fair with both SL/SL and SL/TM $(\kappa=0.26 \pm 0.12)$ and $(\kappa=0.31 \pm 0.12)$.

Table 2 Comparison of paired SL examinations and SL/TM assessment showing proportions of cases with agreement or $\kappa$ values

\begin{tabular}{lcccccc}
\hline & Vascularity total agreement & AC depth (within 10\%) & Bleb height $(\kappa)$ & Wall thickness $(\kappa)$ & Bleb morphology $(\kappa)$ & Bleb leak $(\kappa)$ \\
\hline SL/SL & $75-80 \%$ & $68 \%$ & $0.67( \pm 0.1)$ & $0.63( \pm 0.08)$ & $0.26( \pm 0.12)$ & $0.63( \pm 0.19)$ \\
SL/TM & $63-80 \%$ & $51 \%$ & $0.17( \pm 0.12)$ & $0.39( \pm 0.13)$ & $0.31( \pm 0.12)$ & $0.56( \pm 0.19)$ \\
\hline
\end{tabular}




\section{Bleb leak}

Agreement was similar for both examination techniques $(\kappa=0.63 \pm 0.19$ with SL/SL) and $(\kappa=0.56 \pm 0.19$ with SL/ TM) and ranged from 'moderate' to 'good'. A total of 10 eyes $(25 \%)$ were diagnosed by at least one of the three examiners to have a bleb leak. In three of these, the leak was diagnosed by all three examiners. In three eyes, leak was only diagnosed by TM, not by SL. In three further cases, a leak was identified by one SL observer but not by the other two observers. In the final case, a leak was diagnosed in one SL observer but no entry was made for that patient by the TM observer. In no cases was a leak diagnosed by both SL users but missed by TM.

\section{Microcysts}

Detection of microcysts was not significantly better than chance with both techniques. In $24(60 \%)$ eyes, the examiner using TM scored 'don't know' compared to two $(5 \%)$ and one $(2.5 \%)$ for SL examinations.

\section{Discussion}

Little information exists as to the variability of clinical assessment in eyes with filtering blebs. Paired SL examination provides a 'gold standard' measure for each clinical sign against which alternative equipment can be assessed. High levels of agreement were seen for conjunctival vascularity and anterior chamber depth. In most cases, SL observers agreed within one vascularity score and $10 \%$ of anterior chamber depth estimates. Nominal end points were used for bleb height, wall thickness, bleb morphology and the presence or absence of bleb leak and conjunctival microcysts. We had previously found that the lack of appropriate reference aides, such as standard photographs, made the ordinal scales difficult to use for these signs. Paired SL biomicroscopy achieved good levels of agreement for bleb height, bleb thickness, and bleb leak.

Low levels of agreement were observed for bleb morphology using both examination techniques, despite attempts to simplify the classification to focal, diffuse, or mixed. This may be attributed to preconceived differences between observers as to the definition of each of these bleb morphologies. An alternative explanation may be inherent to the use of the $\kappa$ statistics, as this form of analysis does not take the type of disagreement into consideration. SL observers rarely disagreed between focal and diffuse blebs. Disagreement was usually where one examiner judged a bleb to be either 'focal' or 'diffuse' and the other examiner recorded 'mixed' morphology.

TM has been used for a number of purposes including the monitoring of diabetic retinopathy, strabismus, ${ }^{12}$ oculoplastic disorders, as well as evaluation of postoperative patients ${ }^{13}$ and the coordination of multicentre clinical trials. ${ }^{13}$ With the adoption of new technology in clinical practice, it is important that the clinician has a good understanding of the strengths and weaknesses of the equipment being used. Nitzkin $e t a^{14}$ reported that practitioners with little experience of TM and a poor knowledge of the limitations of the systems in use were more likely to miss important clinical findings. A number of studies have examined the limitations associated with the use of TM in various ophthalmic subspecialties. A prospective interobserver agreement study evaluating the reliability of medium bandwidth teleconferencing equipment in the diagnosis of strabismus revealed good to excellent agreement for horizontal and vertical deviations; however, reduced reliability was noted for the assessment of oblique muscle actions and the detection of small vertical deviations. ${ }^{12}$ Similarly, evaluation of adnexal ophthalmologic examination revealed that teleconsultation with equipment similar to that employed in this study was suitable for assessing congenital or involutional ptosis, but not for less well-defined conditions such as nonspecific ocular pain. ${ }^{15}$ Our study differs from those cited above in that we have assessed the variability of the detection of specific clinical signs rather than the ability of an investigator to assess a given clinical entity.

TM examination provided high levels of agreement for bleb vascularity, anterior chamber depth, and the presence or absence of bleb leak, although the difference in means for SL/TM was slightly larger than for SL/SL. TM assessment was however more variable for bleb height and wall thickness. The optical characteristics of a given clinical sign may determine how well it is detected by the remote observer. Threlkeld $e t a l^{16}$ have suggested that clinical signs with low contrast cues for colour and depth are less reliably observed by TM compared to signs with high contrast cues for colour and depth. This may explain why vascularity and anterior chamber depth, which have high contrast for depth and colour, were more accurately assessed than bleb height and wall thickness, which frequently have relatively low contrast cues. TM achieved good levels of agreement for the detection of bleb leaks. At least one observer diagnosed a bleb leak in $10(25 \%)$ of eyes. Although this proportion is high, the low absolute number reduces the power of this data. However, the TM observer was able to detect bleb leak in all cases where bleb leak was detected by both SL examiners. This suggests that major bleb leaks may be reliably detected with TM. Further investigation with a larger number of bleb leaks is however necessary to substantiate this. 
Implementation of TM for the postoperative review of trabeculectomy cases raises a number of practical points. Firstly, the image acquisition must be optimized. The system employed in this study provides medium-quality video using three ISDN lines delivering up to $384 \mathrm{kbps}$ of data transfer. Further optimization of the video image was obtained by minimizing motion blur, using a dim slit illumination source in association with a diffuse additional illumination source. Secondly, image transmission must be reliable. In a recent review of a teleophthalmology service established between Moorfields Eye Hospital and a regional hospital in South Africa, ${ }^{17}$ there were few reported connection problems over a 1-year period. Thirdly, TM examination must be acceptable to patients. Routine assessment of cataract and trabeculectomy cases on the first postoperative day revealed a high level $(83 \%)$ of patient satisfaction. ${ }^{13}$ Only one patient from the 80 examined found that the experience was unacceptable. In this study, SL image capture and applanation tonometry were performed by a trained nurse. The nurse has also been trained in the removal of releasable sutures and bleb massage.

This study outlines the variability in detecting clinical signs that may be considered important in the evaluation and management of eyes after trabeculectomy. TM examination provides similar levels of variability for bleb vascularity, anterior chamber depth, and bleb leak; however, more subtle signs, such as bleb height and bleb wall thickness, were less well detected by TM compared to SL biomicroscopy. The levels of agreement between observers for bleb morphology and the presence of microcysts were poor for both examination techniques. We propose that remote examination provides a useful mode for assessing trabeculectomized eyes in health care settings where face-to-face evaluation is not deemed practical. Observers should however be aware of the limitations of TM in particular with respect to assessing bleb height and wall thickness.

\section{Acknowledgements}

Funding was provided by the Special trustees of Moorfields Eye Hospital, Wellcome Trust, National Lottery via Fight for Sight and the Keeler Scholarship (Royal College of Ophthalmologists, UK).

\section{References}

1 Picht G, Grehn F. Classification of filtering blebs in trabeculectomy: biomicroscopy and functionality. Curr Opn Ophthalmol 1998; 9: 2-8.

2 Shingleton B. Management of the failing glaucoma filter. Surg Rev 1996; 27: 445-451.

3 Vesti E. Filtering blebs: follow up of trabeculectomy. Ophthalmic Surg 1993; 24: 249-55.

4 Migdal C, Hitchings R. Effect of antiprostaglandins on glaucoma filtration surgery. Trans Ophthalmol Soc UK 1982; 102: 129-132.

5 Mietz H, Arnold G, Kirchhof B, Diestelhorst M, Krieglstein GK. Histopathology of episcleral fibrosis after trabeculectomy with and without mitomycin C. Graefes Arch Clin Exp Ophthalmol 1996; 234: 364-368.

6 Shields MB, Scroggs MW, Sloop CM, Simmons RB. Clinical and histopathologic observations concerning hypotony after trabeculectomy with adjunctive mitomycin C. Am J Ophthalmol 1993; 116: 673-683.

7 Greenfield DS, Liebmann JM, Jee J, Ritch R. Late-onset bleb leaks after glaucoma filtering surgery. Arch Ophthalmol 1998; 116: 443-447.

8 Greenfield DS, Suner IJ, Miller MP, Kangas TA, Palmberg PF, Flynn Jr HW. Endophthalmitis after filtering surgery with mitomycin. Arch Ophthalmol 1996; 114: 943-949.

9 Murdoch I. Telemedicine. Br J Ophthalmol 1999; 83: 1254-1256.

10 Murdoch I, Bainbridge J, Taylor P, Smith L, Burns J, Rendall J. Postoperative evaluation of patients following ophthalmic surgery. J Telemed Telecare 2000; 6 (Suppl 1): S84-S86.

11 Altman DG. Practical Statistics for Medical Research. Chapman \& Hall: London, 1991.

12 Cheung JC, Dick PT, Kraft SP, Yamada J, Macarthur C. Strabismus examination by telemedicine. Ophthalmology 2000; 107: 1999-2005.

13 Kennedy C, Kirwan J, Cook C, Roux P, Stulting A, Murdoch I. Telemedicine techniques can be used to facilitate the conduct of multicentre trials. J Telemed Telecare 2000; 6 : 343-347; discussion 347-349.

14 Nitzkin JL, Zhu N, Marier RL. Reliability of telemedicine examination. Telemed J 1997; 3: 141-157.

15 Rayner S, Beaconsfield M, Kennedy C, Collin R, Taylor P, Murdoch I. Subspecialty adnexal ophthalmological examination using telemedicine. J Telemed Telecare 2001; 7 (Suppl 1): 29-31.

16 Threlkeld AB, Fahd T, Camp M, Johnson MH. Telemedical evaluation of ocular adnexa and anterior segment. $A m$ J Ophthalmol 1999; 127: 464-466.

17 Kennedy C, Van Heerden A, Cook C, Murdoch I. Utilization and practical aspects of tele-ophthalmology between South Africa and the UK. J Telemed Telecare 2001; 7 (Suppl 1): 20-22. 